\title{
Dental practice management and research during the COVID- I 9 pandemic- An overview
}

\begin{abstract}
Dental practice has evolved over time and has been adaptive to the challenges encountered. The current pandemic caused by COVID-19 infection is a new challenge which may induce a paradigm shift in dental practice management. The risk of spread of infection via droplet and air borne routes pose a high risk to the dentist who works in close proximity to patients. This review article introduces the reader to the Sars CoV2 virus, the routes of transmission, clinical presentation of infection, dental practice management tips and implications in dental in vivo research.
\end{abstract}

Keywords: COVID-19 dentistry, research ethics, infection control, Sars CoV2

\author{
Volume 9 Issue 3 - 2020 \\ Sindhu Saeralaathan,' Balaji SK, ${ }^{2}$ Vamsi Lavu ${ }^{3}$ \\ 'Consultant endodontist, SV Dental center, India \\ 2Department of Periodontics, Sri Ramachandra Institute of \\ Higher Education and Research, India \\ ${ }^{3}$ Department, of Periodontics, Sri Ramachandra Institute of \\ Higher Education and Research, India
}

Correspondence: Vamsi Lavu, Professor, Department of Periodontics, Sri Ramachandra Institute of Higher Education and Research, India, Tel +91984I59930I, Email lavu.vamsi@gmail.com

Received: August 23, 2020 | Published: September 10, 2020

\section{Introduction}

A pandemic is defined as "an epidemic occurring worldwide, or over a very wide area, crossing international boundaries and usually affecting a large number of people". ${ }^{1}$ In the early December 2019, people in Wuhan province of China reported with an atypical respiratory disease, which rapidly spread to other provinces. The virus was then named as the severe Acute respiratory syndrome corona virus-2 (SARS-CoV2), since it had a very high homology to the initial SARS $\mathrm{CoV}$ in $2002 .{ }^{2}$ Though it was initially considered to have a zoonotic infection associated from a seafood market in Wuhan province, it was later discovered that the common mode of transmission was human to human leading to a major outbreak. The disease caused by this virus was named as Corona virus disease and was declared as a pandemic by the World Health Organisation (WHO). As of August 2020, more than 22 lakh cases were reported worldwide according to the Center for Systems Science and Engineering (CSSE) at John Hopkins University (JHUoMCr). There has been an exponentially increasing number of patients reported with severe disease who have continued to succumb worldwide. Epidemiological studies show that mortalities are higher in elder population ${ }^{3}$ and the incidence is much lower in children. ${ }^{4-6}$ Current medical management is largely supportive with no targeted therapy available. Several drugs including lopinavir- ritonavir, remdesivir, hydroxychloroquine, and azithromycin are being tested in clinical trials ${ }^{7,8}$ but none of them have been proven to be a therapeutic. More therapies are being tested in clinical trials. In a phased manner which has now reached a stage of human trial in certain countries. A large number of countries have implemented social distancing and lockdown to mitigate further spread of the virus. This article will give a brief description of the corona virus and it implications in dentistry and dental research.

\section{Covid-I 9 viruses and disease pathogenesis}

The Coronavirus $(\mathrm{CoV})$ is a large family of viruses known to cause illnesses ranging from the common cold to acute respiratory tract infection. The severity of the infection may be visible as pneumonia, acute respiratory syndrome, and even death. ${ }^{9}$ Coronaviruses being positive-sense, enveloped, single-stranded RNA viruses, are capable of affecting a wide range of host species. It contains four structural proteins-Spike (S), membrane (M), envelop (E) and nucleocapsid (N). ${ }^{10}$ They have an average size of of $\sim 30 \mathrm{~kb}$ and are divided into four genera; $\alpha, \beta, \gamma$, and $\delta$. Mammals are affected by the $\alpha$ and $\beta$ coronaviruses. Human coronaviruses 229E and NL63 responsible for common cold and croup and belong to $\alpha$ coronavirus family. Angiotensin converting enzyme 2 (ACE2) was identified as a functional receptor for SARS-CoV. ACE2 expression was high in lung, heart, ileum, kidney and bladder. ${ }^{11}$ target needs further investigation. Following the binding of SARS-CoV- 2 to the host protein, the spike protein undergoes protease cleavage.

\section{Factors affecting virus pathogenesis}

Co-morbidities are cardiovascular and cerebrovascular disease as well as diabetes. Several abnormalities also have been observed including cellular immune deficiency, coagulation activation, myocardia injury, hepatic and kidney injury, and secondary bacterial infection. ${ }^{8}$ In the majority of cases of severe disease and death, lymphopenia and sustained inflammation have been recorded. Notably, these observations in COVID-19 patients are similar to those who suffered from severe acute respiratory syndrome (SARS) during the 2003 epidemic. $^{12}$

\section{Modes of spread}

As of April 2020, over 210 countries and territories were affected by the virus, with Europe, the USA, and Iran forming the new cluster of the virus. The presence in different strains of the same virus in different zones of the world might pose a difficulty in analyzing and formulating a treatment plan for the management of the viral disease. ${ }^{10,13}$ Based on the current data, the fatality rate lies around $2 \%$ and an R0 of 3 . Yet a more recent report from the CDC, Atlanta, USA, claims that the $\mathrm{R} 0$ could be as high as 5.7. ${ }^{14}$ Chinese and Indian data also showed a similar trend. The main route of human-to-human transmission is by droplets, which are generated during coughing, talking, or sneezing and are then inhaled by a healthy individual. They can also be 
indirectly transmitted to a person when they contact on surfaces that are touched by a healthy individual who may then touch their nose, mouth, or eyes, allowing the virus entry into the body. As of now, animal-to-human transfer has not yet been confirmed. ${ }^{9}$ Asymptomatic carriers of the virus are at major risk of being superinfectors with this disease, as all those infected may not develop the disease. Fomites are also a common issue in such diseases. ${ }^{43}$ Aerosol-based transmission of the virus has also been confirmed..$^{15}$ Stool-based transmission via the fecal- oral route may also be possible since the SARS-CoV-2 has been found in patient feces. ${ }^{16}$ Some patients with COVID-19 tend to develop diarrhea, which can become a major route of transmission if proper sanitation and personal hygiene needs are not met. There is no evidence currently available to suggest intrauterine vertical transmission of the disease in pregnant women. ${ }^{17}$

\section{Clinical presentation}

\section{Symptoms}

After an incubation period of approximately 5.2 days the symptoms of COVID-19 infection appear. ${ }^{5}$ The period from the onset of symptoms to death ranged between 6 to 41 days with a median of 14 day, which was mainly dependent on the age of the patient and the immune status of the individual. ${ }^{18}$ It was shorter among patients above 70 -years old compared with those under the age of 70 . The most common symptoms are fever, cough, and fatigue. Sputum production, headache, hemoptysis, diarrhea, dyspnea, and lymphopenia may also be seen in a very few early reported patients. ${ }^{18-20}$ In the recent studies it has been stated that sudden loss of smell or taste can also be considered as symptoms of being diseased with COVID-19.21

\section{Clinical signs}

Clinical features presented as pneumonia on a CT chest. There were other features such as anemia, acute respiratory distress syndrome, acute cardiac injury, and presence of grand-glass opacities leading to death. In some cases, the multiple peripheral ground-glass opacities were observed in subpleural regions of both lungs..$^{5,15}$ COVID-19 showed some unique clinical features that include the targeting of the lower airway as evident by upper respiratory tract symptoms like rhinorrhea, sneezing, and sore throat that were characteristic of corona virus family. 22,23

Higher leukocyte numbers, abnormal respiratory findings, and increased levels of plasma pro- inflammatory cytokines are the most common findings in an infected patient. One case report showed a patient at 5 days of fever presented with a cough, coarse breathing sounds of both lungs, and a body temperature of $39.0^{\circ} \mathrm{C}$. The patient's sputum showed positive real-time polymerase chain reaction results that confirmed COVID-19 infection..$^{24}$ The laboratory studies showed leucopenia with leukocyte counts of $2.91 \times 10^{\wedge} 9$ cells/L. $70.0 \% \mathrm{w}$ neutrophils were found in the peripheral smear of the patient. Blood C-reactive protein level was $16.16 \mathrm{mg} / \mathrm{L}$ which is above the range $(0-10 \mathrm{mg} / \mathrm{L})$. High erythrocyte sedimentation rate and D-dimer levels were also observed. ${ }^{5}$ The main pathogenesis of COVID-19 infection as a respiratory system targeting virus was severe pneumonia, anemia, together with the incidence of ground-glass opacities, and acute cardiac injury. ${ }^{25}$ Significantly high blood levels of cytokines and chemokines were noted in patients with COVID-19 infection that included IL1- $\beta$, IL1RA, IL7, IL8, IL9, IL10, basic FGF2, GCSF, GMCSF, IFN $\gamma$, IP10, MCP1, MIP1 $\alpha$, MIP1 $\beta$, PDGFB, TNF $\alpha$, and VEGFA. Some of the severe cases that were admitted to the intensive care unit showed high levels IL2, IL7, IL10, GCSF, IP10, MCP1, MIP1 $\alpha$, and TNF $\alpha$ which are major pro inflammatory cytokines that are reasoned to promote disease severity. ${ }^{3}$

\section{Sars Co V transmission pathway in dentistry}

Dentists, dental hygienists, dental assistants and patients are always at high risk of cross from pathogenic microorganisms and viruses derived from the oral cavity and airways. ${ }^{26} \mathrm{~A}$ study performed on a mannequin fitted with phantom jaws, on a dental chair, showed that the highest levels of aerosol contaminants were found within 60 $\mathrm{cm}$ from the patient's head, mainly on the right arm of the dentist, the mask, and around the nose and eyes. ${ }^{27}$ The aerosol generated by an ultrasonic device can remain suspended in the air for 30 minutes post procedure. ${ }^{28}$ Another in vitro study showed that Sars-CoV-2 maintained viability in the air for at least 3 hours and that its viability half-life was nearly 1 hour. ${ }^{29}$ Additionally, Sars-CoV-2 adheres to various surfaces to a maximum of 9 days; therefore, consider all surfaces and instruments in a dental clinic as potential sources of virus transmission. ${ }^{29,30}$

\section{Dental practice in times of Covid 19}

Since dentists work in close proximity with patients and perform several aerosol generating procedures, the risk of cross infection is high especially during this pandemic. Guidelines have been given by several professional bodies and an adaptation form the ADA guideline has been summarized below.

\section{Dental clinic guideline: (adapted from ada guidelines for dental practice during covid-19)}

a. Maintain ventilation through partial opening of windows and doors.

b. Before start of everyday practice, reduce the bioburden by mopping the floor and clinic area with a regular disinfectant. Do a second round of general clinic area disinfection with water containing $2 \%$ sodium hypochlorite.

c. Use surgical wear in clinic. Avoid general clothing for dentist and assistant.

d. Designate separate donning and doffing area for Personal Protective equipment.

e. Do waste disposal into appropriate color coded bags and hand over to designated bio medical waste disposal authorities.

f. Avoid aerosol generating procedures.

g. Avoid use of closed air circulation systems.

h. Monitor and screen staffs and doctors on a daily basis

i. Work on rotation basis with 2-3 days off in between if more than one doctor/assistantavailable.

j. Train and re-train assistants on patient management and infection control procedures.

k. Make your practice paper free and make digital payment options available.

1. Do not prescribe any medications for patients with COVID-19 symptoms and encourage testing and quarantine if needed. 
m. At the time of closure of practice, do a defogging with hydrogen peroxide plus silver. Particles (Commercially available as Silvox). n. Do a fumigation with $35 \%$ w/w hydrogen peroxide at the time of closure if a procedure has been performed even with minimal aerosol generation.

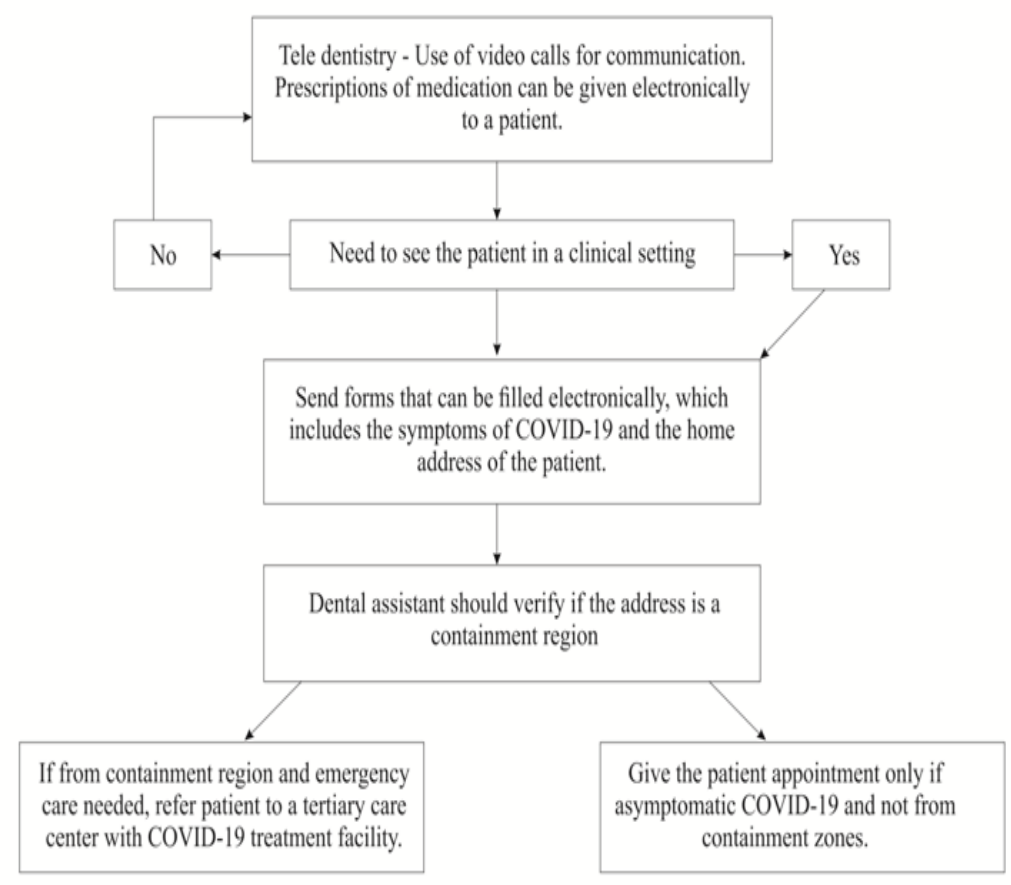

Figure I Suggested protocol of management of patients reporting to a dental practice during a COVID-I9 pandemic- tele-dentistry and triaging.

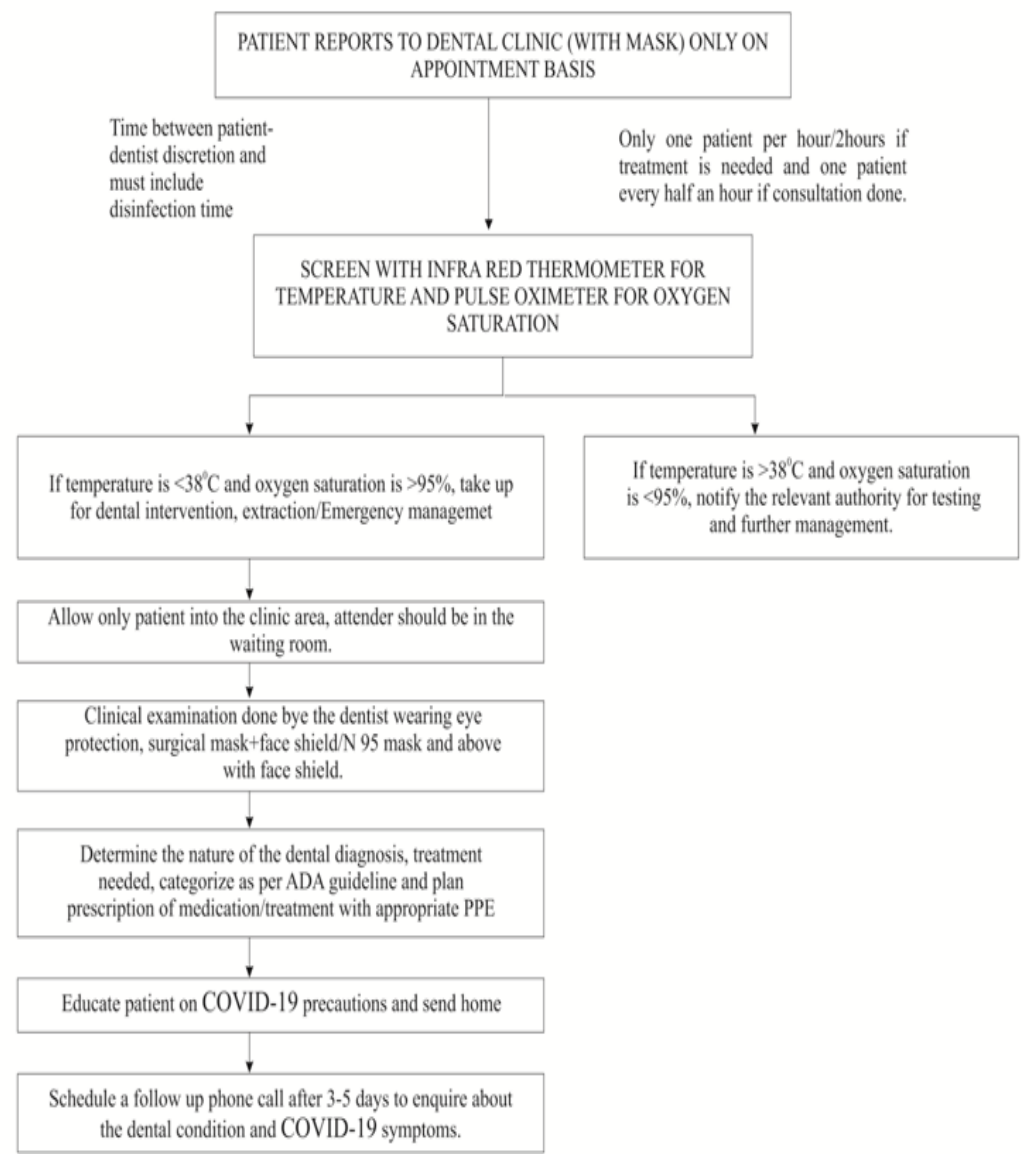

Figure 2 Suggested protocol of management of patients reporting to a dental practice during COVID-I9 pandemic- screening and clinical examination. 
A clinical work flow on managing patients who report to the dentist with the need for emergency treatment is presented below (Figure 1\&2).

The detailed requirements for the dental clinic, sterilization protocols, training of dental health care worker for practice during COVID-19 times should be done as per specifications given by American Dental Association (ADA) and Center For Disease Control (CDC), Atlanta ,USA.

\section{Dental research- ethical issues during covid- 19 pandemic}

In the times of the COVID-19 pandemic, ethics committees have a critical role in decision making and providing approval for research. The WHO has come out with suggestions for ethics committees on norms to be followed for projects based on COVID-19 or drugs related to COVID-19 research. However, specific guidelines for dental research ethics and its challenges are not yet available.

The general guidelines given by WHO for ethical committees to follow during the pandemic include a) minimize the need for face to face interactions with PI b) to provide checklists to enable prioritization of research related to COVID-19 or non COVID-19 related research topics, c) if multicenter trials are to be conducted a document on the safety protocols to be followed at all centres, d) data and biological material transfer agreements and protocols e) insurance terms and conditions is to be provided by the PI to the Ethics committee f) electronic submission of all necessary forms and permission documents, g) time bound review by the select members of committee with support from experts with sound subject knowledge. ${ }^{31}$ Dental in vivo research represents a challenge in terms of the risk posed due to transmission of the virus. In vitro and animal research are however safe and can be carried out with minimal to no risk as long as basics norms of social distancing and personal hygiene measures are followed.

Ethics approval for in vivo research including clinical trial research in dentistry represents a challenge due to multiple factors such as risk of disease transmission, problems in recruiting, exploitation of vulnerable populations (esp) lower socio-economic status through financial incentives, risk benefit ratio may not be favourable and maintenance of privacy/confidentiality. Hence research in dentistry which especially deals with elective surgeries, new dental materials to be tried in vivo for periodontal disease management, educational research in students, cohort studies which are in recruitment phase, dental implant related research may need to be shelved till the time an effective COVID-19 vaccine is developed and administered to the generalpopulation.

\section{Conclusion}

The COVID-19 pandemic is an unexpected challenge to the dental practitioner and will leave its mark on clinical practice management for the years to come. Budding dentists and experienced practitioners need to rework their approach to patient management from the lessons learnt in this period of the pandemic.

\section{Acknowledgments}

None.

\section{Conflicts of interest}

The author declares that there are no conflicts of interest.

\section{References}

1. Last JM. Dictionary of epidemiology. Journal of Epidemiology and Community Health. 1993;47(1):430.

2. Ksiazek TG, Erdman D, Goldsmith CS, et al. A novel coronavirus associated with severe acute respiratory syndrome. $N$ Engl $\mathrm{J} \mathrm{Med}$. 2003;348(20):1953-66

3. Huang C, Wang Y, Li X, et al. Clinical features of patients infected with 2019 novel coronavirus in Wuhan, China. Lancet. 2020.

4. Li L, Ren MJ, Zhang YY. Lung CT image of a confirmed case of the 2019 novel coronavirus (2019- $n \mathrm{CoV}$ ) infected pneumonia (With differential diagnosis of the SARS). Yixue Xinzhi. 2020;30.

5. Li Q, Guan X, Wu P, et al. Early transmission dynamics in Wuhan, China, of novel coronavirus-infected pneumonia. $N$ Engl $\mathrm{J} \mathrm{Med}$. 2020;382(13):1199-207.

6. Jin $\mathrm{Y}-\mathrm{H}$, Cai $\mathrm{L}$, Cheng $\mathrm{ZS}$, et al. A rapid advice guideline for the diagnosis and treatment of 2019 novel coronavirus (2019-nCoV) infected pneumonia (standard version). Mil Med Res. 2020;7(1):4.

7. Wang M, Cao R, Zhang L, et al. Remdesivir and chloroquine effectively inhibit the recently emerged novel coronavirus (2019-nCoV) in vitro. Cell Res. 2020;30(3):269-71.

8. Chen N, Zhou M, Dong $\mathrm{X}$, et al. Epidemiological and clinical characteristics of 99 cases of 2019 novel coronavirus pneumonia in Wuhan, China: a descriptive study. Lancet. 2020;395(10223):507-513.

9. Keni R, Alexander A, Nayak PG, et al. COVID-19: Emergence, Spread, Possible Treatments, and Global Burden. Front Public Heal. 2020;8.

10. Yadav P, Potdar V, Choudhary M, et al. Full-genome sequences of the first two SARS-CoV-2 viruses from India. Indian JMed Res. 2020;151(2):200 209.

11. Sonja A. Rasmussen, MD, MS JCS. Since January 2020 Elsevier has created a COVID-19 resource centre with free information in English and Mandarin on the novel coronavirus COVID-. Ann Oncol. 2020;:19-21.

12. Guo H, Zhou Y, Liu X, et al. The impact of the COVID-19 epidemic on the utilization of emergency dental services. J Dent Sci. 2020;(Xxxx):0-3.

13. Stefanelli P, Faggioni G, Lo Presti A, et al. Whole genome and phylogenetic analysis of two SARS-CoV-2 strains isolated in Italy in January and February 2020: additional clues on multiple introductions and further circulation in Europe. Eurosurveillance. 2020;25(13).

14. Sanche S, Lin YT, Xu C, et al. High Contagiousness and Rapid Spread of Severe Acute Respiratory Syndrome Coronavirus 2. Emerg Infect Dis. 2020;26(7):1470-1477.

15. Rothan HA, Byrareddy SN. The epidemiology and pathogenesis of coronavirus disease (COVID-19) outbreak. $J$ Autoimmun. 2020;109:102433.

16. Yeo C, Kaushal S, Yeo D. Enteric involvement of coronaviruses: is faecal-oral transmission of SARS-CoV-2 possible? Lancet Gastroenterol Hepatol. 2020;5(4):335-337.

17. Chen Y, Guo Y, Pan Y, Zhao ZJ. Structure analysis of the receptor binding of 2019-nCoV. Biochem Biophys Res Commun. 2020;525(1):135-40.

18. Wang $\mathrm{W}, \mathrm{Hu} \mathrm{H}$, Song $\mathrm{L}$. Image of pulmonary and diagnosis of atypicalnovel coronavirus (2019-nCoV) infected pneumonia: case series of 14 patients. Yixue Xinzhi. 2020;30.

19. Wang Y, Lin LK. An advice guideline recommended by central south hospital for the suspected patients of novel coronavirus (2019-nCoV) infected pneumonia and their close contactsas at home quarantine. 2020.

20. Carlos WG, Dela Cruz CS, Cao B, et al. Novel Wuhan (2019-nCoV) Coronavirus. Vol. 201, American journal of respiratory and critical care medicine. United States; 2020. p. 7-8. 
21. Gautier JF, Ravussin Y. A New Symptom of COVID-19: Loss of Taste and Smell. COVID-19 \& Obesity. 2020;28(5):848.

22. Assiri A, Al-Tawfiq JA, Al-Rabeeah AA. Epidemiological, demographic, and clinical characteristics of 47 cases of Middle East respiratory syndrome coronavirus disease from Saudi Arabia: a descriptive study. Lancet Inf Dis. 2013;13:752.

23. Lee $\mathrm{N}$, Hui D, Wu A, et al. A major outbreak of severe acute respiratory syndrome in Hong Kong. N Engl J Med. 2003;348(20):1986-1994.

24. Chen J. Pathogenicity and transmissibility of 2019-nCoV-A quick overview and comparison with other emerging viruses. Microbes Infect. 2020;22(2):69-71.

25. $\mathrm{Xu} \mathrm{Z}$, Shi L, Wang $\mathrm{Y}$, et al. Pathological findings of COVID-19 associated with acute respiratory distress syndrome. Lancet Respir Med. 2020;8(4):420-422.

26. Legnani P, Checchi L, Pelliccioni GA, et al. Atmospheric contamination during dental procedures. Quintessence Int. 1994;25(6):435-439.
27. Veena HR, Mahantesha S, Joseph PA, et al. Dissemination of aerosol and splatter during ultrasonic scaling: a pilot study. J Infect Public Health. 2015;8(3):260-265

28. Consolo U, Bellini P, Bencivenni D, et al. Epidemiological Aspects and Psychological Reactions to COVID-19 of Dental Practitioners in the Northern Italy Districts of Modena and Reggio Emilia. Int J Environ Res Public Health. 2020;17(10).

29. Van Doremalen N, Bushmaker T, Morris DH, et al. Aerosol and Surface Stability of SARS-CoV-2 as Compared with SARS-CoV-1. N Engl J Med. 2020;382(16):1564-1567.

30. Zeng LP, Ge X-Y, Peng C. Cross-neutralization of SARS coronavirusspecific antibodies against bat SARS-like coronaviruses. Sci China Life Sci. 2017;60(12):1399.

31. Nuffield Council on Bioethics. Research in global health emergencies: ethical issues. 2020; P. 276. 